\title{
Análisis de pérdidas económicas por enfermedades en el sistema de producción de búfalos en el departamento de Córdoba, Colombia
}

\author{
Analysis of economic losses due to diseases in the buffalo production \\ system in the department of Córdoba, Colombia
}

\author{
Antonio María Martínez Reina ${ }^{1}$ \\ Erly Luisana Carrascal Triana ${ }^{2}$ \\ Matiluz Doria Ramos ${ }^{3}$ \\ Juan Andrés García Jiménez ${ }^{4}$ \\ Jorge Armando Mejía Luquez ${ }^{5}$ \\ Erika Patricia Caicedo Carrascal ${ }^{6}$
}

DOI: https://doi.org/10.19053/01228420.v18.n2.2021.12582

RESUMEN: El objetivo de este trabajo fue analizar las pérdidas económicas por efecto de las enfermedades en el sistema de producción de búfalos en el departamento de Córdoba (Colombia). Se partió de la información generada en la línea de base, pero con un tratamiento con mayor especificidad en el tema de enfermedades. La información se originó de dos fuentes: una encuesta aplicada a 30 productores y un taller participativo, el método análisis correspondió al de criterios múltiples a través de un consenso con productores. Los datos se organizaron en archivos planos, se calcularon las estadísticas descriptivas y cálculos porcentuales dentro de las diferentes categorías de animales, como de las variables: producción y tasas de mortalidad, disminución de producción por causa de enfermedades. Los resultados mostraron que las enfermedades relacionadas con hemoparásitos y endoparásitos incurren en mayor número de productos para el tratamiento; en el caso del carbón sintomático solo se hace tratamiento preventivo que consiste en la vacuna. Se concluye que el componente mayor del costo es por concepto de medicamentos con un $68 \%$ del total calculado, seguido por las pérdidas bucerros con un $11 \%$ y por último, la producción de carne con un $6 \%$. En total las pérdidas se estimaron en 30.235.048 pesos COP. Nuevas estrategias de manejo sanitario deben ser implementadas para reducir el gasto generado por las enfermedades en búfalos.

PALABRAS CLAVE: Retorno económico, eficiencia, tratamientos, producción, ventas, rentabilidad, ganancias.
ABSTRACT: The objective of this work was to analyze the economic losses due to diseases in the buffalo production system in the department of Córdoba in Colombia. It was based on the information generated in the baseline, but with a treatment with greater specificity in the subject of diseases. The information originated from two sources: A survey on producers applied to 30 producers and a participatory workshop, using the multiple criteria analysis method through a consensus with producers. The data were organized in flat files, descriptive statistics and percentage calculations were calculated within the different categories of animals as well as the variables: production and mortality rates, decreased production due to disease. The results show that diseases related to hemoparasites and endoparasites incur a greater number of products for treatment, in the case of symptomatic charcoal, only preventive treatment consisting of the vaccine is done. In this context, it is concluded that the largest component of the cost is for medicines with $68 \%$ of the calculated total, followed by calf losses with $11 \%$ and finally meat production with $6 \%$. In total the losses were estimated at 30.235.048 COP pesos. New sanitary management strategies should be implemented to reduce the expense generated by buffalo diseases.

KEYWORDS: Economic return, efficiency, treatments, production, sales, profitability, profits. org/10.19053/01228420.v18.n2.2021.12582 


\section{Introducción}

Al revisar los datos del inventario de búfalos del Instituto Colombiano Agropecuario ICA, se pudo establecer que la producción de Bufalos a nivel nacional como en el departamento de Córdoba, han presentado tasas de crecimiento positivas en los últimos cinco años, siendo mayor el crecimiento del número de cabezas a nivel nacional con $8,5 \%$, en tanto que para el departamento de Córdoba, registra un crecimiento de 7,8 en el periodo comprendido entre 2016 y 2020 . Con relación al número de predios dedicados a la producción de esta especie el crecimiento fue mayor para el caso del departamento de Córdoba con 10,9 \% para el mismo periodo analizado. Es de anotar que en el último año el departamento de Córdoba presentó un decrecimiento tanto el número de cabezas como en el de predios de $-5 \%$ y $-1,2 \%$ respectivamente (ICA, 2021). No obstante, la actividad bufalina se considera promisoria como alternativa de inversión en el subsector pecuario.

La importancia relativa del sistema de producción de búfalos con relación a las variables número de cabezas ubica al departamento de Córdoba en el primer lugar con 86.263 cabezas en 330 predios. Le sigue del departamento de Antioquia con 54.638 cabezas en 412 predio, es de anotar que supera al departamento de Córdoba en el número de predios (ICA, 2021).

Dominguez et al. (2013) señalan que por el búfalo ser un bóvido puede padecer muchas enfermedades de los bovinos como la leptospirosis, la brucelosis, la tuberculosis y la colibacilosis. Una vez se reconoce que existen enfermedades y que se manifiestan desde el punto de vista clínico, condición corporal, bajas en producción, se requiere calcular las perdidas por la presencia de enfermedades con el fin de contribuir a la solución mediante la valoración de los daños económicos en los sistemas de producción de búfalos.

A pesar de que el productor afronta las pérdidas e incurre en costos por prevención y tratamiento de las enfermedades en los búfalos, en pocas oportunidades se analizan las pérdidas económicas y no se lleva control estricto de los rubros por estos conceptos. Como resultado del análisis de la información se identificaron los conceptos de las pérdidas por enfermedades producidas por hemoparásitos y endoparásitos para determinar las implicaciones económicas en la unidad productiva.

Una de las preocupaciones en el sistema productivo de búfalos son las pérdidas que ocurren por la mortalidad en los diferentes grupos de animales, bajas tanto en la producción de carne y leche, como en los parámetros reproductivos y costos de los tratamientos de las enfermedades que atacan a la especie bufalina (Singh, 2012). Los componentes de los costos están asociados con la enfermedad y la productividad, reflejados en la producción láctea, la producción de crías y costos asociados con las estrategias de control, (Claros et al., 2005). Por otra parte, es conveniente determinar los costos de la 
prevención, tratamiento y manejo de las enfermedades ya sean preventivas o para el tratamiento de enfermedades (Mathew \& Menon, 2008).

Dado lo nueva que es la especie en los sistemas de producción pecuaria en el país y en departamento de Córdoba, la literatura sobre evaluación económica de enfermedades en la especie bufalina no es abundante y por tanto se considera este trabajo pionero en esta línea de investigación, la cual tiene como objetivo estimar las pérdidas por enfermedades en los sistemas de producción de búfalos del departamento de Córdoba por ser uno de los más representativos de población de la especie en Colombia.

\section{Materiales y Métodos}

El trabajo se realizó en 30 unidades productivas de búfalos en el departamento de Córdoba, se tomó como referencia una unidad productiva de 250 hectáreas con 450 animales compuesto por diferentes categorías y estados productivos. El 45\% del total corresponde a 200 hembras en producción bajo el supuesto que no hay ningún disturbio o enfermedad que limite su producción, el 39\% lo constituyen machos cebados con pesos promedio vivo de 468 $\mathrm{Kg}$ y un $15 \%$ restante corresponde a la cría de bucerros destetos con pesos vivos de $170 \mathrm{~kg}$.

En un estudio previo realizado por la Corporación Colombiana de Investigación Agropecuaria - AGROSAVIA en el 2019, se generó información general sobre las diferentes enfermedades del sistema de producción de búfalos manifestados en los principales signos y síntomas como son: Decaimiento con un $21 \%$, inapetencia $19 \%$, tristeza $10 \%$, ojos llorosos, diarrea y anemia fue reportado en un $7 \%$ respectivamente, fiebre $6 \%$, pérdida de peso $4 \%$ de la muestra encuestada. En bajo porcentaje (3\%) se presenta rigidez muscular, cojera y trastornos respiratorios. Además, algunos productores mencionaron que sus búfalos presentaban signos de renguera, papada inflamada, erizados, bajo crecimiento, parálisis y lesiones de la cavidad abdominal.

El origen de la información para definir las enfermedades a considerar en este estudio, fue la información de 30 fincas de productores quienes no especificaron realmente a qué enfermedad presentaban los animales, razón por la cual condujo a la necesidad de realizar una descripción detallada de cada uno de los síntomas, dando como resultado que los principales disturbios del hato se debían a la presencia de endoparásitos, hemoparásitos y carbón sintomático y sobre estas enfermedades o agentes causantes de enfermedades se realizó la evaluación de pérdidas por enfermedades.

El primer paso consistió en identificar las restricciones que afectan los sistemas de producción con sus causas y efectos. Una vez identificados, se clasificaron y priorizaron por mayor incidencia las que se atribuyen a enfermedades infecciosas y parasitarias y en segundo lugar se cuantificaron las 
bajas en la producción ya sea en carne o en leche, los cuales son elementos para considerar para el cálculo de pérdidas económicas (González, 2007).

\section{Perdidas en producción de leche}

Para la producción de leche se tomó una cantidad de 200 hembras en diferentes etapas productivas (paridas, preñadas y secas), se calculó que un 20\% del total de hembras se encontraban en ordeño, con un periodo de lactancia de 270 días y una producción total promedio de 41.091 litros. Una vez se obtuvo el resultado de la disminución de litros de leche/año, se multiplicó por su precio que para el año 2019 fue de 1.415 pesos COP/Litro en promedio y de esta forma se expresa en términos monetarios el total de la pérdida por concepto de leche.

El cálculo de las perdidas en leche por efecto de las enfermedades se puede formalizar así:

$$
\text { PerLech }=[(\text { Litros año })-(\text { Litros año } \times 0,06)] \times P
$$

Donde:

PerLech $=$ Pérdidas de leche

Litros año = Número total de litros de leche producidos en un año.

$\mathrm{P}=$ Precio promedio de litro de leche.

El resultado de la ecuación es el total de perdidas monetarias por causa de las enfermedades en búfalos.

\section{Pérdidas en producción de carne}

Para el cálculo de la pérdidas por carne, se tuvo en cuenta la ecuación 2 , considerando la producción total de kilos de carne del animal en pie, además las variables como: el peso vivo promedio de un búfalo cebado, (468 kg/animal). Se estimó aproximadamente un 39\% de participación del total de hato para un total de 176 animales en las 250 hectáreas de la finca de referencia. Las cantidades de carne producidas en un año fueron de $12373 \mathrm{~kg}$ de animal en pie. Se consideró una pérdida del $20 \%$ en carne. Al valor de carne obtenido se resta el $20 \%$ que genera el total de $\mathrm{kg}$ de carne que se dejan de producir por efecto de las enfermedades. Con un precio promedio de venta de 3.800 pesos COP/kg. Se calculó el valor monetario de la perdida.

Los cálculos se presentan bajo la siguiente formalización:

$$
\text { PerCarne }=[(\text { Kilos año })-(\text { Kilos año } \times 0,20)] \times P
$$

Donde:

PerCarne $=$ Pérdidas de leche

Kilos de carne año = Número total de kilos de carne producidos en un año. $\mathrm{P}=$ Precio promedio de kilo de carne. 
El resultado de la ecuación es el total de pérdidas monetarias en el componente de carne por causa de las enfermedades en búfalos.

\section{Pérdidas en producción de bucerros}

Para el cálculo se utilizó el método de los dos componentes anteriores, se parte de un número de 23 bucerros que producen un total de 3910 kilos de animal en pie en las 250 hectáreas en un año. Además, se tuvo en cuenta variables como: el peso promedio del bucerro desteto (170 kilos promedio). Se consideró una pérdida del $20 \%$ en el total de producción. Al total de la producción de kilos de bucerro producidos se le restó el $20 \%$ como pérdida, que son las cantidades que dejan de producir por efecto de las enfermedades. Con un precio promedio de venta de 3.200 pesos COP/Kg. Se calculó el valor monetario de la perdida bajo la siguiente formalización:

$$
\text { Perbucerro }=[(\text { Kilosbu año })-(\text { Kilosbu año } \times 0,20)] \times P
$$

Donde:

Perbucerro $=$ Pérdidas de kilos de bucerro

Kilosbuaño = Número total de kilos de bucerro producidos en un año.

$\mathrm{P}=$ Precio promedio de kilo de bucerro.

El resultado de la ecuación es el total de pérdidas monetarias en el componente de kilos de bucerro en pie por causa de las enfermedades en búfalos.

Una vez analizados los tres componentes se hace un análisis comparativo de las dos situaciones: los ingresos sin presencia de enfermedades y con presencia de enfermedades.

\section{Gastos por tratamientos}

El otro componente lo constituye los gastos por tratamiento para el manejo de enfermedades, el cual se obtuvo por medio de la aplicación de 13 encuestas y entrevistas personalizadas a los encargados de la producción como propietarios, médicos veterinarios y mayordomos, se tuvo como criterio que llevaran registros y estuvieran en disposición de compartir la información. Se priorizaron las enfermedades con mayor incidencia en el hato en el departamento de Córdoba , las cuales son las causadas por hemoparásitos, endoparásitos y carbón sintomático, se obtuvieron datos de las dosis aplicadas y valor de cada una de ellas, de acuerdo con el precio pagado por los productores. Tomando el valor de los tratamientos y el costo de la mano de obra de las tres enfermedades se llegó al cálculo total de los costos de enfermedades en los sistemas de producción de búfalos.

Como complemento al ejercicio se hicieron los análisis comparativos para dos situaciones, una con tratamiento de enfermedades y una convencional 
con algunos tratamientos mínimos, la comparación se hizo con base en los retornos económicos estableciendo las diferencias en cada caso, las técnicas empleadas se basan en las aplicadas por Martinez (2020).

\section{Resultados y Discusión}

Las principales enfermedades de los búfalos de acuerdo con el criterio del productor están relacionadas con la presencia de hemoparásitos en un 59\% de la muestra encuestada, seguido por septicemia con un $14 \%$ de la muestra, enfermedades respiratorias en un $11 \%$, carbón sintomático con $8 \%$ de la muestra y en baja proporción (3\%) Endoparasitosis, Clostridiosis y Mastitis. La mayoría de las enfermedades están relacionadas con: Hemoparasitosis, Endoparasitosis y Carbón Sintomático

Con este inventario se realizaron los cálculos por pérdidas, para el caso de la leche se estimó una baja del $20 \%$ que, de un total de 40 vacas en producción, 23 machos cebados y 26 bucerros destetos.

Para el cálculo de las perdidas físicas al año, se tomaron los porcentajes de disminución de cada uno en las diferentes categorías. Los cuales se presentan en la Tabla 1.

TABLA 1. Perdidas por cantidades físicas dejadas de producir por enfermedades en búfalos en un año.

\begin{tabular}{|l|c|c|c|}
\hline \multirow{2}{*}{ Componente } & \multirow{2}{*}{ Pérdidas en físicas/año } & Pérdidas en dinero & Pérdidas en dinero \\
\cline { 3 - 4 } & & US\$* & \$ COP: \\
\hline Leche $(\mathrm{Lt})$ & 2.465 & 979 & 3.488 .651 \\
\hline Carne $(\mathrm{Kg})$ & 587 & 527 & 1.876 .800 \\
\hline Bucerros $(\mathrm{Kg})$ & 884 & 943 & 3.359 .200 \\
\hline Total & 3.936 & 2.449 & 8.724 .651 \\
\hline
\end{tabular}

*Tasa Representativa del mercado TRM 3,561.9111 de marzo de 2021

Se presentan disminuciones en la producción en los tres componentes: Carne, leche y bucerros, en cantidades físicas, en el caso de la leche por efectos de las enfermedades, se dejaron de producir 2.465 litros al año, es de anotar que se trabajó con un periodo de lactancia de 270 días, la cantidad de dinero dejada de percibir el productor fue de US\$979 por año. En pesos colombianos corresponden a $\$ 3.488 .651$ pesos.

Para el caso de la carne se dejan de producir $587 \mathrm{~kg}$ de carne para un valor de US\$527 por año, en pesos colombianos es de 1876800 pesos por año y por ultimo los bucerros que se dejaron de producir fueron 884 kilos de animal en pie para un valor en dólares de US $\$ 943$, en pesos colombianos es de $\$ 3.359 .200$ pesos. 
El valor pagado por los tratamientos para las enfermedades: Hemoparasitosis, Endoparasitosis y Carbón Sintomático, donde, se tuvo en cuenta la dosis del tratamiento y el valor de la mano de obra para aplicarlo, se presenta en la Tabla 2.

TABLA 2. Valor del costo para tratamiento de enfermedades en búfalos en el departamento de Córdoba.

\begin{tabular}{|l|c|c|c|}
\hline Enfermedad & $\begin{array}{c}\text { Costo del Tratamiento } \\
\text { COP. }\end{array}$ & $\begin{array}{c}\text { Costo del Tratamiento } \\
\text { US } \$^{*}\end{array}$ & Par \\
\hline Hemoparasitosis & 7.680 .816 & 2.156 & 37 \\
\hline Endoparasitosis & 6.907 .032 & 1.939 & 33 \\
\hline Carbón Sintomático & 6.055 .830 & 1.700 & 29 \\
\hline Total, Enfermedades & 20.643 .678 & 5.796 & 100 \\
\hline
\end{tabular}

*Tasa Representativa del mercado TRM 3,561.91 11 de marzo de 2021

De acuerdo con los datos de animales en producción de todo el hato con las participaciones porcentuales en cada caso, son 99 animales afectados, correspondiente al $22 \%$ del total de hato (450 animales). Se aprecia que las variaciones entre los diferentes componentes son mínimos, con un coeficiente de variación del $8 \%$, es decir, que se aleja muy poco del promedio de los tres componentes de las tres enfermedades en estudio. Al analizar con más profundidad la composición de estos, se puede a concluir que la mano de obra tiene un mayor peso en la composición del costo.

El análisis conjunto de los tres elementos que componen el análisis; perdidas por bajas en la producción de leche, perdidas por las bajas en la producción de carne y las perdidas en la producción de terneros, sumado a los costos de los tratamientos se pueden apreciar en la Tabla 3.

TABLA 3. Cálculos totales de pérdidas por enfermedades en búfalos en el departamento de Córdoba 2020.

\begin{tabular}{|l|c|c|c|}
\hline Concepto de la pérdida & Valor en cantidades & Valor en Pesos COP. & Valor Dólares US\$* \\
\hline Pérdidas por leche & 3.078 & 4.355 .370 & 1.223 \\
\hline Pérdidas por Carne & 587 & 1.876 .800 & 527 \\
\hline Pérdidas por bucerros & 884 & 3.359 .200 & 943 \\
\hline Costos enfermedades & & 20.643 .678 & 5.796 \\
\hline Total & & 30.235 .048 & 8.488 \\
\hline
\end{tabular}

*Tasa Representativa del mercado TRM 3,561.91 11 de marzo de 2021

En la Tabla 3, se muestran los componentes que constituyen las pérdidas por presencia de enfermedades causadas por hemoparásitos, endoparásitos y carbón sintomático, tiene una mayor participación el tratamiento de las enfermedades, supera a las pérdidas en conjunto de la producción de leche, 
carne y bucerros que en total suman $32 \%$, en tanto que lo relacionado con costos de medicinas y mano de obra participan con el $68 \%$ del total.

Los análisis y cálculos anteriores permiten definir que tomando en conjunto las tres enfermedades, sus tratamientos y las bajas producidas, se aprecia un impacto económico alto, si se tiene en cuenta que lo que deja de ganar el productor por esta causa asciende anualmente a 30.235 .048 pesos COP correspondiente a US\$8488.

Desde otra perspectiva, los efectos de las enfermedades y su tratamiento se consideraron dos situaciones, una con presencia de las tres enfermedades y sus respectivos tratamientos y la otra en forma convencional tal como realiza el manejo sanitario el productor, lo cual se puede apreciar en la Tabla 4.

TABLA 4. Análisis comparativo del sistema de producción de búfalos con tratamiento de enfermedades y manejo convencional 2020.

\begin{tabular}{|l|c|c|c|}
\hline Indicador & Convencional & Enfermedades & Diferencia \\
\hline Labores & 60.375 .000 & 38.508 .000 & 21.867 .000 \\
\hline Tratamiento enfermedades & 2.148 .800 & 20.643 .678 & -18.494 .878 \\
\hline Alimentación & 2.975 .000 & 2.975 .000 & 0,00 \\
\hline Instalaciones & 1.293 .500 & 1.293 .500 & 0,00 \\
\hline Costos directos & 66.792 .300 & 63.420 .178 & 3.372 .122 \\
\hline Costos indirectos & 10.515 .808 & 10.183 .228 & 332.580 \\
\hline Costos Totales & $\mathbf{7 7 . 3 0 8 . 1 0 8}$ & $\mathbf{7 3 . 6 0 3 . 4 0 6}$ & 3.704 .702 \\
\hline Ingresos leche & 58.143 .765 & 54.655 .790 & 3.487 .975 \\
\hline Ingresos carne & 40.903 .200 & 34.766 .200 & 6.137 .000 \\
\hline Ingresos bucerros & 14.144 .000 & 11.315 .200 & 2.828 .800 \\
\hline Ingresos Totales & $\mathbf{1 1 3 . 1 9 0 . 9 6 5}$ & $\mathbf{1 0 0 . 7 3 7 . 1 9 0}$ & 12.453 .775 \\
\hline Ingreso Neto & 35.882 .857 & 27.133 .784 & 8.749 .073 \\
\hline Costo Litro leche & 950 & 1034 & -84 \\
\hline Costo kilo carne & 2.552 & 2.776 & -225 \\
\hline Costo kilo bucerro & 2.149 & 2.338 & -189 \\
\hline Rentabilidad & 46 & 37 & 9,6 \\
\hline B/C & 1,46 & 1,37 & 0,1 \\
\hline
\end{tabular}

Fuente: Cálculos con base en trabajo de campo Agrosavia 2020

Los resultados permiten apreciar que la presencia de enfermedades se constituyen un factor negativo que afecta la producción de búfalos por bajas en la producción tanto de carne como de leche siendo mayor el costo por concepto de tratamiento de las enfermedades con un porcentaje de participación mayor en el total de los costos (68\%) si bien se presentan pérdidas en carne y leche manifestadas en las cantidades que se dejan de producir las cuales participan en un $32 \%$ del total de los costos. De todas maneras, con presencia de enfermedades la producción presenta indicadores de retorno 
positivos aun con presencia de enfermedades, pero estos presentan bajas en la medida que aumentan las enfermedades.

Con relación a la presencia de enfermedades de los hatos bufalinos en el departamento de Córdoba, el trabajo permitió poner en evidencia que las mayores afectaciones económicas se relacionan con los aumentos en los costos y las cantidades de dinero que se dejan de percibir como consecuencia de las bajas en la producción de carne, leche y bucerros o crías que tienen como consecuencia afectaciones negativas en los retornos económicos. Igualmente se atribuyen las bajas de los ingresos netos al aumento de los costos en los tratamientos. Por otra parte, se evidencia que los ingresos son mayores cuando no hay afectaciones por enfermedades que se refleja en bajas en el ingreso neto de la unidad productiva en presencia de enfermedades.

Al comparar los resultados obtenidos en un estudio sobre enfermedades de búfalos en la india por Singh (2014), se encontró en primer lugar que utilizan los mismos parámetros y comparando las perdidas por leche en el caso de este estudio participan en un $72 \%$ del total en tanto que el estudio de Singh (2014) lo hace en un 49,12\% del total de pérdidas, esto se explica porque el número de litros que se dejan de producir por año son mayor para el caso del departamento de Córdoba ( 3078 litros al año) en tanto que para el estudio de referencia es de 625 litros por año. De todas maneras, los dos estudios muestran los efectos negativos de la presencia de enfermedades en los hatos bufalinos debido a las bajas en la producción de leche en este caso.

Los análisis se dirigieron a evaluar los diferentes componentes del costo clasificados en costos directos e indirectos, se tuvo en cuenta cuando hay presencia de enfermedades, los resultados muestran que los costos directos participan con el $63 \%$ en tanto que los costos indirectos fueron de $37 \%$ Es de anotar que en este estudio se incluyó la administración dentro de los costos indirectos. Estos resultados se comparan con el estudio realizado por Dewia et al 2020 para medir las pérdidas económicas por la enfermedad de Murra causada por el protozoo flagelado Trypanosoma evansi. Sumba Timur, Nusa Tenggara Timur-Indonesia con encuestas a 30 unidades productivas al igual que este trabajo, encontró que los costos indirectos eran del $46 \%$ en tanto que los costos directos del $37 \%$ costos de administración y monitoreo el $17 \%$. Dentro de los costos indirectos el tratamiento preventivo participó con el $40 \%$. Además, en este rubro contemplaron las pérdidas por muertes, bajas en la producción y costos del tratamiento una vez hay presencia de la enfermedad, situación similar a la adoptada en el presente trabajo. Lo anterior deja claro que los métodos usados tanto en este trabajo como el de Dewia (2020) para estimar las perdidas por enfermedades son consistentes y permiten entender mejor la realidad frente a las perdidas por enfermedades.

La estimación de las perdidas por enfermedades en búfalos para el departamento de Córdoba siguió una metodología similar a la de Mathew \& Menon 
(2008) para estimar perdidas por fiebre aftosa en rebaños de bovinos y fúfalos. Los cálculos permiten estimar las perdidas por disminución en la producción de leche del $18 \%$ en promedio. En el caso del departamento de Córdoba, se encontró que registró un $20 \%$ de disminución en la producción de leche con una perdida en dinero de 4.355.370 COP. Lo anterior permite inferir que si se previenen las enfermedades las perdidas podrían disminuir en los dos casos.

Es de anotar que dado a lo novedosa que es esta especie en Colombia comparada con los bovinos que llevan más de 500 años, la importación de los búfalos data de 1967 de Trinidad y Tobago, la literatura acerca de la evaluación económica del impacto de enfermedades de los búfalos es escasa y se considera un trabajo pionero para esta especie por esta razón, no se referencian resultados en este tema y se espera continuar avanzando tanto en cobertura como en profundidad temática en esta materia, se resalta la adaptación y generación de técnicas de cálculos y análisis de pérdidas por enfermedades de los búfalos en el departamento de Córdoba en este trabajo.

\section{Conclusiones}

Considerando los sistemas de producción de búfalos como de doble propósito se aprecia tres salidas: la producción de leche, la producción de carne de macho cebado y los bucerros destetos. Se aprecia que están inclinados más a producción de leche tal vez por el flujo de caja que genera y hace sostenible al sistema de producción.

Los cálculos y estimaciones permiten determinar los efectos negativos de la presencia de enfermedades en disminuciones del 32\% con mayor afectación en la producción de leche. Por su parte, los costos por tratamientos de las enfermedades que incluyen el valor del medicamento y la mano de obra para su aplicación se calculan en un $68 \%$ del total de los costos.

Los análisis y cálculos realizados permiten concluir que tomando en conjunto las tres enfermedades, sus tratamientos y las bajas producidas, el impacto económico es alto si se tiene en cuenta que lo que deja de ganar el productor por esta causa asciende anualmente a 15.224.571 pesos COP por año.

La producción animal de un hato se ve afectada por la presencia de enfermedades reflejadas en disminuciones en las cantidades físicas tanto de leche, carne y la producción de crías. Por una parte, la producción de leche baja por que algunas hembras dejan de producir o por reducción en la producción de litros de leche por día. Las pérdidas en la producción de carne se dan por pérdida de peso y en algunos casos por muertes, se estima que las tasas de mortalidad son del $6 \%$. 
Los indicadores de retorno son afectados cuando hay presencia de enfermedades en general en todo el rebaño, en este caso los costos aumentaron tanto por efectos del valor de las medicinas como por la mano de obra adicional para su tratamiento, esto hace que por ejemplo los costos unitarios de producción sean más altos con presencia de enfermedades en los tres productos que genera el sistema de producción: leche, carne y bucerros. Esto como ya se explicó se debe a los costos de los tratamientos. Tal vez con unas medidas preventivas y buen manejo animal, se podría disminuir este costo y hacer mucho más rentable el negocio.

\section{Agradecimientos}

Al Ministerio de Agricultura y Desarrollo Rural (MADR), por la financiación del proyecto ID: 1001394, que dio origen a esta investigación, a la Corporación Colombiana de Investigación Agropecuaria AGROSAVIA. A los productores de búfalos por la paciencia en el suministro de la información.

\section{Referencias}

Arenas, N., \& Moreno, V., (2016) Estudio económico de la infección por brucella abortus en ganado bovino de la región del sumapaz, Colombia. Rev Med Vet Zoot. 63(3), septiembre - diciembre $218-228$.

Claros, A., Camacho A., \& González, A., (2005). Pérdidas económicas por brucelosis bovina en un hato lechero (Provincia Andrés Ibáñez, departamento de Santa Cruz).Tesis de pregrado. Santa Cruz de la Sierra, Bolivia. Universidad Autónoma Gabriel René Moreno.

Dewia, R., Damajantib, A., Wardhanac, A., Mulasihd, O., Steenevelde, P., \& Hogeveen H. (2020) The Economic Losses of Surra Outbreak in Sumba Timur, Nusa Tenggara Timur-Indonesia. Tropical Animal Science Journal. 43(1):77-85 DOI: https:// doi.org/10.5398/tasj.2020.43.1.77

Dominguez, G., Romero, D., Martínez, D \& Garcia, S., (2013) Los búfalos de agua y las enfermedades infecciosas. Revista de divulgación científica y tecnológica de la universidad veracruzana. Volumen XXVI Número 2. Disponible en: https:// www.uv.mx/cienciahombre/revistae7vol 26num2/articulos/bufalos.html

Forero, J., Garay, L., Barberi, F., Ramírez, C., Suárez, D. \& Gómez, R. (2013). La eficiencia económica de los grandes, medianos y pequeños productores agrícolas colombianos. En Reflexiones sobre la ruralidad y el territorio en Colombia. Problemáticas y retos actuales. Bogotá D.C.: Corporación Nuevo Arco Iris.

González. R., Pérez. M., \& Brito.S., (2007) Fasciolosis bovina. Evaluación de las principales pérdidas provocadas en una empresa ganadera. Rev. Salud Anim. 29(3): 167-175 Disponible en: http:// scielo.sld.cu/pdf/rsa/v29n3/rsa07307.pdf

ICA. (2020) Censo Pecuario año 2020. Disponible en: https://www.ica.gov.co/ areas/pecuaria/servicios/epidemiologia-veterinaria/censos-2016/censo-2018. aspx 
ICA. (2021) Censo pecuario años 2016 al 2020. Disponible en: https://www.ica. gov.co/areas/pecuaria/servicios/epidemiologia-veterinaria/censos-2016/censo-2018.aspx

Martinez, A., Doria, M., Herrera, N., Garcia, J,.\& Salcedo, E,. (2020). Caracterización técnica y económica del sistema de producción de búfalos Bubalus bubalis en el departamento de Córdoba de Colombia. Revista Archivos de Zootecnia. 69(286) Doi: https:// doi.org/10.21071/az.v69i268.5392

Mathew, L., \& Menon, R. (2008). Economic Impact of FMD in Chazhoor Panchayath, Veterinary World. 1(1):5-6. Disponible en: https://www.researchgate.net/ publication/49607926_Economic_Impact_of_FMD_in_Chazhoor_Panchayath

Singh, B., Shiv, P., Rama, V. \& Sinha, D., (2014) Estimation of economic losses due to foot and mouth disease in India. Indian Journal of Animal Sciences. 83 (9): 964-970, September 2013/Article. Disponible en: www.veterinaryworld.org/ Vol.7/April-2014/1.pdf. 